\title{
IMAGINÁRIO: DERIVAÇÕES DE MÉTODOS NO BRASIL ${ }^{1}$
}

\section{IMAGINARY: DERIVATIONS OF METHODS IN BRAZIL}

\author{
Danielle Perin Rocha Pitta ${ }^{2}$
}

\begin{abstract}
Resumo
O Imaginário na perspectiva do antropólogo Gilbert Durand, desde 1974, tem tido grande repercussão no Brasil. Teoria e métodos são cada vez mais utilizados nos diversos campos do saber, seja o AT-9 (Arquétipo teste de 9 elementos do psicólogo Yves Durand), seja a mitodologia (mitanálise e mitocrítica). A diversidade de interesses provocou a criação de derivações de métodos, principalmente em relação ao AT-9, como o ATL-9 direcionado para arquitetura e urbanismo. Os dados estatísticos expostos dão uma idéia da importância destes estudos na Academia brasileira.
\end{abstract}

Palavras-chave : Imaginário. Gilbert Durand. Mitodologia. AT-9. Brasil.

\begin{abstract}
The Imaginary in the perspective of the anthropologist Gilbert Durand, since 1974, has had great repercussion in Brazil. Theory and methods are increasingly used in the various fields of knowledge, whether the AT-9 (psychologist Yves Durand's 9-element test archetype), or the mitodology (mitanálise and mitocrítica). The diversity of interests has led to the creation of method derivations, especially in relation to AT-9 such as the ATL-9 for architecture and urban planning. The statistical data presented give an idea of the importance of these studies in the Brazilian Academy.
\end{abstract}

Keywords: Imaginary. Gilbert Durand. Mitodologia. AT-9. Brazil.

Considera-se o imaginário, aqui, na perspectiva de Gilbert Durand, lembrando que duas hipóteses centrais orientam a metodologia por ele elaborada:

$1^{\circ}$ - não existe corte entre os cenários significativos das antigas mitologias e o agenciamento moderno dos relatos culturais: literatura, belas artes,

\footnotetext{
${ }^{1}$ Parte destes dados foram publicados nos Anais do II Congresso Internacional da rede CRI2I (Centre de Recherches Internationales sur l'Imaginaire): a Teoria Geral do Imaginário 50 anos depois: conceitos, noções, metáforas - 2015

${ }^{2}$ Professora aposentada associada do PPGA da Universidade Federal de Pernambuco. Membro do bureau diretor do Centre de Recherche International sur l'imaginaire (CRI2I) sediado em Cluj, Romênia. Vice-presidente da Association des amis de Gilbert Durand (AAGD), sediada em Chambéry, França. Presidente da Associação Ylê Seti do Imaginário - Recife. 
ideologias e histórias...; (...) hipótese da existência de uma continuidade entre o imaginário mítico e a positividade histórica;

$2^{\circ}$ - os comportamentos concretos dos homens, e mais precisamente o comportamento histórico, repetem com timidez (...) os cenários e as situações dramáticas dos grandes mitos (DURAND,1979).

Logo, para a compreensão em profundidade, seja de textos, de obras de arte ou do cotidiano, é necessário ter acesso ao nível mítico a eles subjacentes.

Importante lembrar também que esta teoria permitiu o desenvolvimento de métodos de pesquisa suprindo uma necessidade importante quando se trata de lidar com o símbolo.

Vale ressaltar que, fora da França, seu país de origem, os países que mais têm se dedicado à teoria e aos métodos do Imaginário de Gilbert Durand, são o Brasil e a Romênia. Pesquisas são neles desenvolvidas em vários campos do saber.

No que diz respeito ao Brasil, desde 1973 o Centro de Estudos sobre o Imaginário (Fundação Joaquim Nabuco), hoje Núcleo Interdisciplinar de Pesquisas sobre o Imaginário (NIPI-UFPE), desenvolve estudos comparados de grupos culturais. Foram assim estudadas as culturas Fulni-ô (grupo indígena de Pernambuco), Afro-brasileiras (adeptos das religiões afro no Recife), do nordeste e sul do Brasil (estudantes universitários). Outros temas tratados foram e são : arte como expressão cultural ${ }^{3}$; imaginário da $3^{\mathrm{a}}$ idade (estudos encabeçados por Altair Macedo Lahud Loureiro - Brasília), discurso político; saúde e cultura (imaginário dos doadores de rins, das dimensões culturais no tratamento do câncer bucomaxilofacial), no jogo de RPG, entre tantos outros.

Todos utilizam métodos decorrentes da teoria de G. Durand: uma mitodologia composta da aplicação do teste AT-9 e suas derivações, da mitocrítica e da mitanálise. Lembramos que a ambição da mitanálise é de

decifrar as grandes orientações míticas de momentos históricos e culturais coletivos... Esta mitanálise tenta vislumbrar, atrás dos exemplos da Mitocrítica textual, o jogo dinâmico pelo qual um agrupamento humano ligado por um destino cultural agencia seus temores e seus desejos, suas metas e suas visões do mundo, para constituir a alma pela qual se identifica

\footnotetext{
${ }^{3}$ Cf. os temas dos Ciclos de Estudo de Recife http://www.yle-seti-imaginario.org/home/artigo/Histrico/34

Téssera | Uberlândia, MG | v.1 | n.1 | p.154-172 | jul./dez. 2018 | ISSN 2595-8925 
e sobrevive como tal através dos avatares e das vicissitudes do devir (DURAND, 1979, p. 8).

Os grupos de estudo mais relacionados com educação, e são os mais numerosos, tratam das "mediações simbólicas" em organizações educativas ${ }^{4}$, e tiveram início graças ao trabalho pioneiro do CICE (Centro de Estudos do Imaginário, Cultura e Educação da USP) com José Carlos de Paula Carvalho e Maria Cecilia Sanchez Teixeira principalmente. Na Bahia encontra-se o Kawé tratando da dimensão pedagógica dos cultos afro-brasileiros (Ruy Povoas, Consuelo Oliveira Santos) ${ }^{5}$; no Rio de Janeiro o LISE (Laboratório do Imaginário Social e Educação - da UFRJ); no Maranhão, João de Deus Vieira Barros da UFMA ${ }^{6}$; e o grupo Cultura, Imaginário, Memória, Narrativa e Educação - CIMNE, coordenado pela professora Iduína Mont'Alverne Chaves - UFF.

Em História tem-se o importante trabalho realizado pelo Departamento de Ciências das Religiões (UFPB) com os grupos Videlicet - Religiões, desenvolvendo entre outros o estudo da bacia semântica ligada à inquisição (tese de doutorado do professor Carlos André Cavalcanti) ${ }^{7}$ : estudos sobre Intolerância, Diversidade e Imaginário.

Em Literatura destacam-se Maria Teresa Strôngoli (PUC-SP) e Sebastien Joachim (UFPE) com vários livros publicados e atualmente o Núcleo de Pesquisa NELIM - Núcleo de Ecolinguística e Imaginário ${ }^{8}$ coordenado por Elza Kioko Nakayama Nenoki do Couto que entre outras publicações, apresenta a "Antropologia do Imaginário, ecolinguística e metáfora", organizado por Elza Kioko / Ema Marta Dunck-Cintra / Lorena Araújo de Oliveira Borges (Thesaurus - 2014).

O Nordeste, o Sudeste, o Sul e o Centro-Oeste do Brasil muito têm contribuído para a divulgação dos estudos do imaginário na Literatura. O POEIMA - Grupo de Pesquisa Poéticas e Imaginário, coordenado pelas pesquisadoras Enivalda Nunes Freitas e Souza (UFU) e Elzimar Fernanda Nunes Ribeiro (UFU) é de configuração interdisciplinar e promove o estudo sistemático do imaginário em textos literários e artísticos. O corpus

\footnotetext{
${ }^{4}$ Cf. a Revista de Educação Pública de 1994

${ }^{5}$ Núcleo de Estudos Afro-Baianos Regionais - Kàwé http://www.uesc.br/nucleos/kawe/index.php?item=conteudo apresentacao.php

${ }^{6}$ http://arteculturaeimaginario.blogspot.com.br

7 Cavalcanti, C. A.: Doutorado em História. Universidade Federal de Pernambuco, UFPE, Brasil. Título: 0 Imaginário da Inquisição: Desmitologização de Valores no Tribunal do Santo Ofício, no Direito Inquisitorial e nas Narrativas do Medo de Bruxa (Portugal e Brasil, 1536-1821), 2001

8 http://www.nelim.letras.ufg.br 
literário é formado por obras de escritores representativos da poesia, da prosa e do teatro. $\mathrm{O}$ método de abordagem e reflexão é o mitocrítico, de Gilbert Durand, e consiste na investigação das imagens, dos símbolos e dos mitos das produções artísticas, congregando as hermenêuticas psicanalítica, linguística, histórica, filosófica, bem como as teorias da literatura e da arte. O POEIMA tem como objetivo sedimentar a linha de pesquisa "Poéticas do Texto Literário: Cultura e Representação", do Programa de Pós-Graduação em Estudos Literários (cursos de Mestrado e Doutorado) da Universidade Federal de Uberlândia, fornecendo aporte teórico aos professores e alunos em suas atividades de ensino e pesquisa. São integrantes desse grupo professores da História, da Filosofia, da Psicologia e das Artes. A principal produção intelectual do POEIMA pode ser conferida em seus três livros publicados: Reflexos e sombras: arquétipos e mitos na literatura (2010); Entre o mito, o sagrado e o poético: ecos de uma sinfonia (2013); Poesia com deuses: estudos de Hídrias, de Dora Ferreira da Silva (2016) ${ }^{9}$.

Entre os projetos inspirados pelos Estudos do Imaginário na Faculdade de Letras da Universidade Federal de Goiás está a Biblioteca Bilíngue de Literatura Infantil e Juvenil Libras / Português (Bibliolibras), que reúne livros audiovisuais em um espaço virtual e acessível, o site: www.bibliolibras.com.br. O formato desses livros, apresentados em libras e português (escrito e oral), permite que sejam usados por quaisquer crianças (surdas, ouvintes, deficientes visuais e com outras formas de deficiência) em telas de notebooks, tablets ou celulares, facilitando o acesso a textos de literatura Infantil e Juvenil, especialmente aos contos de tradição oral ${ }^{10}$.

Outro grupo de relevância nos estudos do imaginário na literatura está sediado na Universidade Estadual da Paraíba, o CEPESI - Centro Paraibano de Estudos do Imaginário, coordenado por Maria Goretti Ribeiro. De igual importância é o ITESI - Grupo de Pesquisa Itinerários Interdisciplinares em Estudos Sobre o Imaginário, coordenado por Geam Karlo Gomes, da Universidade de Pernambuco.

$\mathrm{Na}$ Universidade Federal do Rio Grande (RS), o Grupo de Pesquisa "Literatura,

\footnotetext{
${ }^{9}$ Todas essas publicações do POEIMA foram financiadas pela FAPEMIG - Fundação de Amparo à Pesquisa do Estado de Minas Gerais.

${ }^{10}$ Criada em 2017 com recursos do Fundo de Cultura, a Bibliolibras alcançou professores e alunos surdos em todo o país, conforme depoimentos de visitantes (endereço: www.bibliolibras.com.br).
} 
Imaginário e Poéticas da Contemporaneidade", liderado por Mairim Linck Piva, com a colaboração de Cláudia Mentz Martins, tem organizado eventos e orientado pesquisas sobre o imaginário sul-riograndense e de outras culturas.

Pesquisadores do POEIMA, do CEPESI, do ITESI e do "Literatura, Imaginário e Poéticas da Contemporaneidade" integram o GT "Imaginário, representações literárias e deslocamentos culturais", da ANPOLL - Associação Nacional de Pós-Graduação e Pesquisa em Letras e Linguística.

Quanto à Arquitetura: o método por mim proposto em 1993, o ATL-9 (Arquétipo teste do lugar) derivado do AT-9 de Yves Durand, tem sido utilizado na École d'Architecture de Grenoble, tendo por produtos o Diplôme de Sophie Chambe sobre o vilarejo Real de Catorce no México (1997), e o espetáculo "Noto" sobre a Sicília, em Cenografia (2000), por Tania Pitta. Também a tese de doutorado da mesma utilizou o teste, o que permitiu "passeios" por cidades de três países: "Promenades Imaginales dans le creux des villes contemporaines. De l'Imprévisible beauté de la forme. Noto, Belleville, Morro da Conceição" (Paris V - 2009).

Trata-se de desenhar e dispor em um mapa de um bairro ou de uma região, os 9 elementos do teste, isto aplicado a um número representativo de habitantes do lugar, com a finalidade de se obter um mapa sensível do mesmo. O novo método foi batizado por Tania Pitta de ATL-9 (arquétipo teste do lugar de 9 elementos) (Pitta, 2015) ${ }^{11}$. Anteriormente o mesmo teste foi aplicado no estudo de um colégio, os arquétipos (positivos ou negativos) sendo representados e situados na sala da diretoria, nas salas de aula, no pátio do recreio, nos banheiros etc. (GARCEZ, 2000).

Em Comunicação, temos a preciosa contribuição de Muniz Sodré que, com seu pertencimento simultâneo a duas culturas, propõe indispensáveis "Jogos Extremos do Espírito" e "Pensar Nagô". Juremir Machado da Silva, da PUC-RS com as pesquisas em Cultura midiática e tecnologias do imaginário, seus vários livros publicados a este respeito, e Ana Tais Portanova Barros que lidera o Imaginalis - Grupo de Estudos sobre Comunicação e Imaginário / CNPq / UFRGS.

Tema relevante ainda, o estudo da vivência de mulheres em meio Rural (Flávia Maia Guimarães): A luta pela terra: Imaginário e Gênero (1998) - orientação de Neide Miele -

\footnotetext{
${ }^{11} \mathrm{Cf}$. Também a sua tese de doutorado op. cit. 
UFPB) através da aplicação de uma derivação do AT-9 (Arquétipo teste de 9 elementos) por ela desenvolvida: uma aplicação coletiva com as mulheres do campo, não desenhado, mas sim montado à partir de materiais diversos (isopor, pedras, plantas, etc.).

Veremos a seguir adaptações e variações efetuadas sobre as propostas metodológicas durandianas. Muitas são relativas ao teste AT-9 de Yves Durand, que consiste na elaboração de um microuniverso mítico.

Foi feita em 1975, pelo NIPI da UFPE, uma padronização (inédita) do teste AT-9, propondo novas categorias para a sua interpretação, como por exemplo, acrescentar a opção "família" entre os adjuvantes (Rocha Pitta, D.P., 1975) ${ }^{12}$, que não constava no contexto francês.

Dependendo da especificidade do objeto de estudo, o AT-9, inicialmente aplicado pelo seu criador de maneira individual, foi adaptado, em várias ocasiões, para aplicação coletiva. Assim é que Flávia Maia Guimarães, como já citado, para estudar o imaginário de mulheres em área rural, analfabetas, pediu para um grupo pré-selecionado de agricultoras para que, em conjunto, construíssem o microuniverso proposto. A história a ser contada também foi feita de forma coletiva. Assim pôde ser observado o semblante da angústia na figura antropomorfa, frequentemente representando o patrão, o dono da terra, ligada ao poder; as formas de defesa escolhidas, etc. Outra aplicação coletiva foi feita em relação ao estudo de um grupo de pichadores alunos de uma escola de periferia do Recife: uma parede do pátio foi disponibilizada assim como latas de spray de tintas para responderem coletivamente ao teste (GARCEZ, 2000).

Em atenção à obra de M. Maffesoli, e à sua observação de que o cotidiano institui o sentido, e notando o quanto, no cotidiano, a sexualidade é tema das conversas, foi empreendido um estudo que viria a elaborar uma nova epistemologia relativa às categorias sexuais ou de gênero.

Em conferência apresentada na XV Semana de Humanidades (UFRN) em Maio de 2007, intitulada: O corpo inserido em diversas lógicas culturais: uma poética da sexualidade $^{13}$, e antes no Congresso de Americanistas em Sevilha, eu colocava que, após trabalho de campo realizado no Recife, puderam ser observadas, no cotidiano da cidade e

\footnotetext{
12 Inédito

${ }^{13}$ https://periodicos.ufrn.br/bagoas/article/view/2283 (aceessado 28/7/2018)

Téssera | Uberlândia, MG | v.1 | n.1 | p.154-172 | jul./dez. 2018 | ISSN 2595-8925
} 
não na academia, a existência de 17 categorias sexuais (por um grupo de lésbicas em seus discursos, reunido em 2004). Existe aí uma lógica bem menos excludente que a lógica binária. Ora, a proposta que então fazíamos e que continuamos fazendo, em adequação com a teoria geral do imaginário e com as práticas do cotidiano, é considerar a existência não mais de categorias relativas à sexualidade ou a questões de gênero, mas de polarizações criando assim uma dinâmica específica. A sexualidade se atualiza então entre os dois polos feminino e masculino. Tendo em vista a noção de "trajeto antropológico": "o incessante intercâmbio existente, a nível do imaginário, entre as pulsões subjetivas e assimiladoras e as intimações objetivas emanando do meio cósmico e social" (DURAND, 1975, p. 38), propomos a sua operacionalização em relação à sexualidade. O trajeto sexual seria: o incessante intercambio existente, a nível do imaginário, entre as pulsões femininas e as pulsões masculinas, emanando do corpo físico e social, e expressando-se pela corporeidade, ou pelo cogito corporal.

Paralelismos:

$$
\mathrm{M}=\text { místico } \quad \mathrm{H}=\text { heroico }
$$

\section{TRAJETO SEXUAL}

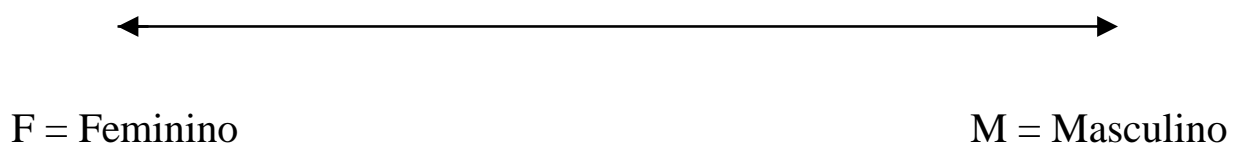

Nesta perspectiva, a sexualidade sendo uma dinâmica, não há mais possibilidade de exclusão de uma ou outra categoria. Assim como a identidade, esta dinâmica varia no tempo: cada cultura, assim como cada indivíduo, pode se locomover no seu próprio eixo, construir o seu trajeto, viver seu próprio destino sendo atraído mais ou menos por um dos polos segundo as circunstâncias. No plano mítico, esta concepção da sexualidade é bem ilustrada 
pelas imagens dos deuses Shiva ou Oxumare que conjugam as várias dimensões da sexualidade.

Bem dizia Gilbert Durand, no século passado, que "as conjunturas epistemológicas do século XX exigem pois uma mudança de direção da crítica como do método ». O que dizer do século XXI ? Pode-se dizer que a mudança ocorreu: Aí estão as grandes redes de pesquisas internacionais como a RIIR (Rede Ibero-americana de Pesquisa sobre Imaginário e Representações), o CRI2I (Centre de Recherche International sur l'Imaginaire), a Association des amis de Gilbert Durand. Somente no Brasil, registrados no CNPQ, são 78 grupos de pesquisa e 411 linhas de pesquisa ${ }^{14}$.

Como bem dizia G. Durand: "A um discurso sobre o método, totalmente ultrapassado, deve suceder um discurso sobre as entidades plurais que tecem o novo campo do saber e do agir. (Durand, 1960), e que "toda antropologia autêntica deve começar pela mitologia”.

A Declaração de Veneza vem complementar:

O estudo conjunto da natureza e do imaginário, do universo e do homem, poderia assim nos aproximar mais do real e nos permitir enfrentar melhor os diferentes desafios de nossa época" ${ }^{15}$.

\section{Referências}

CAVALCANTI, C. A. O Imaginário da Inquisição: Desmitologização de Valores no Tribunal do Santo Ofício, no Direito Inquisitorial e nas Narrativas do Medo de Bruxa (Portugal e Brasil, 1536-1821). Doutorado em História. Universidade Federal de Pernambuco. Brasil. 2001

DURAND, G. As estruturas antropológicas do imaginário - Martins Fontes - 1997

DURAND, G. L'anthropologie et les structures du complexe - Sociétés 2007/4 (n 98) https://www.cairn.info/revue-societes-2007-4-page-7.htm. Acesso em 28 de Julho 2018) DURAND, G. L'Âme tigrée, Paris, Denoël, 1980.

\footnotetext{
${ }^{14}$ Dados coletados no site do CNPQ em 26/7/2018. Estão relacionados em anexo para se ter uma idéia de sua repartição por Estados e por disciplinas, mesmo que sejam interdisciplinares.

15 DECLARAÇÃO DE VENEZA. Comunicado final do Colóquio "A Ciência diante das Fronteiras do Conhecimento" - Veneza, 7 de março de 1986 
DURAND, G. L'imaginaire. Essai sur les sciences et la philosophie de l'image. Paris: Hatier, 1994

DURAND, G. Figures mythiques et visages de l'œuvre. De la mythocritique à la mythanalyse, Paris, Berg International, 1979

GARCEZ, Rita. O muro do Cemitério. Polissemia de uma escola. Dissertação de Mestrado em Antropologia - PPGA/UFPE - 2000

MAFFESOLI, Michel. La Conquête du présent. Pour une sociologie de la vie quotidienne, Paris, PUF 1979

OLIVEIRA, Elda Rizzo de. Comunicação mediática, modelo biomédico e curas mediúnicas - Cronos, Natal-RN, v. 7, n. 1, p. 105-126, jan./jun. 2006

OLIVEIRA, E. R. Representações de doença e estratégias de cura: os magos da ciência e os cientistas da magia. In: BOTAZZO, C.; TORRES, S. Ciências sociais e saúde bucal: questões e perspectivas. São Paulo. Ed. UNESP, 1998

PITTA, Tania. In Discutindo o Imaginário: olhares multidisciplinares; Ed. UFPE - 2015 PORTANOVA BARROS, A. T. M. (Org.) Anais II Congresso do CRI2i. A Teoria Geral do Imaginário 50 anos depois: conceitos, noções, metáforas. Porto Alegre. Brasil, 2015 ROCHA PITTA, D. Fractalité et nouvelles constellations mythiques au Brésil, Loxias 2 (janv. 2004), mis en ligne le 15 janvier 2004, URL : http://revel.unice.fr/loxias/index.html?id=872. Acesso em 28 de julho de 2018)

ROCHA PITTA, D. O corpo inserido em diversas lógicas culturais: uma poética da sexualidade. Bagoas v. 2, n. $03-2008$

ROCHA PITTA D.P.: Para uma arquitetura sensível. Revista de Antropologia (PPGA/UFPE), UFPE Recife, 1998.

RUBIO, K. O atleta e o mito do herói: o imaginário esportivo contemporâneo Paperback - 2001

SODRÉ, Muniz. Jogos extremos do espírito, Rocco Ed., 1994

SODRÉ, Muniz. Pensar Nagô. Ed. Vozes, 2017

THOMAS, Jöel (org.). Introduction aux méthodologies de l'imaginaire. Paris: Ellipses, 1998

TURCHI, Maria Zaíra. Literatura e Antropologia do Imaginário. Brasília: UnB, 2003. 
VOTRE, S. (org.) : Imaginário \& representações sociais em: educação física, esporte e lazer. Rio de Janeiro: Ed. Gama Filho, 2001

WUNENBURGER , J. J. Imaginaires du Politique. Ellipses, Coll. Philo, 2001

\section{GRUPOS DE PESQUISA CADASTRADOS NO CNPQ ${ }^{16}$}

(Conselho Nacional de Desenvolvimento Científico e Tecnológico)

1. Antropologia das Paisagens: memórias e imaginários na Amazônia. Instituição: UFPA

Líder(es): Flávio Leonel Abreu da Silveira, Rosangela Marques de Britto

Área: Antropologia

2. Antropologia, Imaginário e Educação. Instituição: UFJF

Líder(es): Julvan Moreira de Oliveira

Área: Educação

3. ARTCRI - Grupo de Pesquisa em Artes Cênicas, Imagem e Imaginário. Instituição: UFBA

Líder(es): Catarina Sant'Anna

Área: Artes

4. Arte, cultura e imaginário. Instituição: UNISA

Líder(es): Maria Auxiliadora Fontana Baseio

Área: Artes

5. Arte, Cultura e Imaginário na Educação - GSACI. Instituição: UFMA

Líder(es): João de Deus Vieira Barros, Josenildo Campos Brussio

Área: Educação

6. Artes do Corpo: memória, imagem e imaginário. Instituição:UEFS

Líder(es): Ana Rita Queiroz Ferraz, Luís Vítor Castro Júnior Área: História

7. Centro Interdisciplinar de Estudos e Pesquisas do Imaginário Social. Instituição: UNIR

\footnotetext{
16 Publicado em parte in Estado de la investigación en Iberoamérica en torno a los imaginarios y las representaciones sociales RIIR - 2018 
Líder(es): Valdir Aparecido de Souza, Edinaldo Bezerra de Freitas Área: Antropologia

8. Corpo, cultura e imaginário social. Instituição: UFRJ

Líder(es): Simone Freitas Chaves

Área: Educação Física

9. Cultura, Imaginário, Memória, Narrativa, e Educação (CIMNE). Instituição: UFF Líder(es): Iduina Edite Mont Alverne Braun Chaves Área: Educação

10. Diásporas, Memórias e Imaginários nas Literaturas das Américas. Instituição: UFRJ Líder(es): Ana Maria Lisboa de Mello, Ricardo Postal Área: Letras

11. Dramaturgias Contemporâneas: Percursos entre Adaptação, Gênero, História e Imaginário. Instituição: FURG

Líder(es):Valter Henrique de Castro Fritsch

Área: Letras

12. Gabinete de Estudos da Informação e do Imaginário (GEDII). Instituição: UFMG

Líder(es): Claudio Paixão Anastácio de Paula

Eliane Pawlowski de Oliveira Araújo

Área: Ciência da Informação

13. Educação estética e imaginário. Instituição: UPF

Líder(es): Graciela René Ormezzano, Gérson Luís Werlang

Área: Artes

14. GEIPaT - Grupo de Estudos de Imaginário, Paisagem e Transculturalidade. Instituição: UFG

Líder(es): Valéria Cristina Pereira da Silva, Givaldo Ferreira Corcinio Junior Área: Geografia

15. GEPAI - Grupo de Estudo e Pesquisa em Antropologia do Imaginário. Instituição: UFPB

Líder(es): Eunice Simões Lins Gomes, Leyla Thays Brito da Silva Área: Teologia

16. Geracionalidade, imaginário e violência. Instituição: UCB/DF 
Líder(es): Vicente de Paula Faleiros

Área: Psicologia

17. GERIS - Grupo de Estudos sobre Representação Social, Imaginário, Memória e Intervenção Profissional. Instituição: UEL

Líder(es): Antonio Geraldo Magalhães Gomes Pires, Jeane Barcelos Soriano

Área: Educação Física

18. Gestão pública educacional, imaginário e cultura. Instituição: UFPE

Líder(es): Evson Malaquias de Moraes Santos

Área: Educação

19. Grupo de Estudo e Pesquisa em Enfermagem sobre o Quotidiano, Imaginário, Saúde e Família - GEPEQUISF. Instituição: UEM

Líder(es): Cremilde Aparecida Trindade Radovanovic

Área: Enfermagem

20. Grupo de Estudos e Pesquisas em Educação e Imaginário Social. Instituição: UFSM Líder(es): Valeska Maria Fortes de Oliveira, Lúcia Maria Vaz Peres Área: Educação

21. Grupo de Pesquisa Cultura, Imaginário e Educação - GPCIE. Instituição: UFPEL Líder(es): Cláudio Baptista Carle, Angelita Hentges Área: Antropologia

22. Grupo de Estudos sobre transgressões, imagens e imaginários. Instituição: UFMG Líder(es): Ida Lucia Machado, Emília Mendes Lopes Área: Lingüística

23. Grupo de Pesquisa e de Estudos Memória e Imaginário das Vozes e Escrituras das Culturas Populares. Instituição: UFPB

Líder(es): Beliza Aurea de Arruda Mello

Área: Lingüística

24. Grupo de Pesquisa Imagem e Imaginários. Instituição: PUCRS

Líder(es): Juliana Tonin

Área: Comunicação

25. Imaginário e Cotidiano. Instituição: UNISUL

Líder(es): Heloisa Juncklaus Preis Moraes 
Área: Letras

26. Grupo de Tecnologias do Imaginário. Instituição: PUCRS

Líder(es): Juremir Machado da Silva, Francisco Eduardo Menezes Martins

Área: Comunicação

27. Grupo Interdisciplinar de Pesquisa e Extensão em Contemporaneidade, Imaginário e Teatralidade. Instituição: UFBA

Líder(es): Luiz Claudio Cajaiba Soares, Ciane Fernandes

Área: Artes

28. História, cultura e imaginário. Instituição: UFRGS

Líder(es): Carla Simone Rodeghero, Miriam de Souza Rossini

Área: História

29. Identidade, Memória, Imaginário. Instituição: UNIP

Líder(es): Silvana Dudonis Vitorelo Iizuka

Área: Arquitetura e Urbanismo

30. Imaginalis - Grupo de Estudos sobre Comunicação e Imaginário. Instituição: UFRGS Líder(es): Ana Tais Martins Portanova Barros, Jean-Jacques Wunenburger Área: Comunicação

31. Imaginar. Grupo de pesquisas sobre imaginário, educação e formação de professores. Instituição: UNIOESTE

Líder(es): Andrea Cristina Martelli, Tânia Maria Rechia Schroeder

Área: Educação

32. Imaginário e Cultura no Ocidente Medieval. Instituição: UFAM

Líder(es): Sinval Carlos Mello Gonçalves

Área: História

33. Imaginário e Informação: Estudos Culturais e Comparativos. Instituição: UFF Líder(es): Rosa Inês de Novais Cordeiro

Área: Ciência da Informação

34. Imaginário Educação e Memória. Instituição: UFPEL

Líder(es): Lúcia Maria Vaz Peres, Andrisa Kemel Zanella

Área: Educação

35. Imaginário Mítico na Literatura e em outras Artes. Instituição: UEPB 
Líder(es): Maria Goretti Ribeiro, Francisca Zuleide Duarte de Souza Área: Letras

36. Imaginário Religioso Brasileiro. Instituição: PUC/SP

Líder(es): Enio Jose da Costa Brito, Claudio Santana Pimentel Área: Teologia

37. Imaginário Social da Saúde, Esporte e Lazer. Instituição: UGF

Líder(es): Nilda Teves Ferreira, Monique Ribeiro de Assis Área: Educação Física

38. Imaginário Tecnológico. Instituição: UFRJ

Líder(es): Ieda Tucherman, Cecilia Carrossini Bezerra Cavalcanti Área: Comunicação

39. Imaginário, Símbolo e Mito. Instituição: UFVJM

Líder(es): Roberto Antônio Penedo do Amaral Área: Filosofia

40. Imaginários na Comunicação Visual (Imagicom). Instituição: UEL Líder(es): André Azevedo da Fonseca Área: Comunicação

41. ÍMAN - Imagem, Mito e Imaginário nas Artes da Cena. Instituição: UFG Líder(es): Alexandre Silva Nunes, Luciana de Fátima Rocha Pereira de Lyra Área: Artes

42. LIMES - Literatura e Imaginário, Marginalidade, Estética e Sociedade. Instituição: UFG

Líder(es): Alexander Meireles da Silva, Silvana Augusta Barbosa Carrijo Área: Letras

43. Laboratório do Imaginário Social e Educação. Instituição: UFRJ Líder(es): Nyrma Souza Nunes de Azevedo, Reuber Gerbassi Scofano Área: Educação

44. LAPIIS - Laboratório de Pesquisa em Infância, Imaginário e Subjetividades. Instituição: UFF

Líder(es): Andréa Cardoso Reis, Ana Lúcia Lima da Costa Área: Educação 
45. Literatura de Massa: os romances sentimentais e o imaginário feminino. Institui.: UECE

Líder(es): Roberta Manuela Barros de Andrade, Erotilde Honorio Silva

Área: Sociologia

46. Literatura e Diversidade Cultural: imaginário, linguagens e imagens. Instituição: UNEB

Líder(es): Andréa do Nascimento Mascarenhas Silva, Valdete da Macena Pardinho Área: Letras

47. Literatura, História e Imaginário. Instituição: URI - Universidade Regional Integrada do Alto Uruguai e das Missões

Líder(es): Denise Almeida Silva

Área: Letras

48. Literatura, História e Teorias do Imaginário. Instituição: PUCRS

Líder(es): Ana Maria Lisboa de Mello

Área: Letras

49. Literatura, Imaginário e Poéticas da Contemporaneidade. Instituição: FURG Universidade Federal do Rio Grande

Líder(es): Mairim Linck Piva, Claudia Mentz Martins

Área: Letras

50. Memória e Imaginário nas Literaturas Brasileira e Africanas. Instituição: UFRPE Líder(es): Sávio Roberto Fonsêca de Freitas, Iêdo de Oliveira Paes

Área: Letras

51. Memórias do ABC: Memória e Imaginário em novos contextos da Comunicação Instituição: USCS

Líder(es): Priscila Ferreira Perazzo

Área: Comunicação

52. Mídia e Estudos do Imaginário. Instituição: UNIP

Líder(es): Malena Segura Contrera, Jorge Miklos

Área: Comunicação

53. Nas fronteiras da Linguagem, a indistinção: práticas significativas, imaginário e vida social (da violência e da resistência). Instituição: UNICAMP 
Líder(es): Eni de Lourdes Puccinelli Orlandi

Área: Lingüística

54. NELIM- Núcleo de Estudos de Ecolinguística e Imaginário. Instituição: UFG

Líder(es): Elza Kioko Nakayama Nenoki do Couto, Maria Thereza Guimarães Strongoli Área: Lingüística

55. Núcleo de Estudos em Estética e Política dos Imaginários (NEPI). Instituição: UNILA

Líder(es): Dinaldo Sepúlveda Almendra Filho

Área: Artes

56. Núcleo de Estudos em Neorregionalismo, Imaginário e Narratividade. Instituição: UESPI

Líder(es): Herasmo Braga de Oliveira Brito

Área: Letras

57. Núcleo de Estudos sobre Imaginário e Linguagem - NEIL. Instituição: UESB

Líder(es): Clédson Luciano Miranda dos Santos, José Carlos da Silva Simplício Área: Artes

58. Núcleo de Pesquisa e Estudos sobre Enfermagem, Quotidiano, Imaginário, Saúde e Família de Santa Catarina - NUPEQUIS-FAM -SC. Instituição: UFSC

Líder(es): Rosane Goncalves Nitschke, Ana Maria Farias da Silva Área: Enfermagem

59. Núcleo Interdisciplinar de Estudos sobre o Imaginário. Instituição: UFPE

Líder(es): Danielle Perin Rocha Pitta, Maria das Vitorias Negreiros do Amaral Área: Antropologia

60. O Imaginário da Exclusão - grande imprensa e protestos, Brasil, 2014. Instituição: USP

Líder(es): Marília Pacheco Fiorillo

Área: Comunicação

61. O imaginário poético hispano-americano. Instituição: UFRJ

Líder(es): Mariluci da Cunha Guberman

Área: Letras

62. OIKOUMENE - Núcleo de Estudos e Pesquisas em Religião, Cultura e Imaginário 
Instituição: UFAM

Líder(es): Marilina C. Oliveira Bessa Serra Pinto, Marco Aurélio Coelho de Paiva Área: Filosofia

63. POEIMA - Grupo de Pesquisa Poéticas e Imaginário. Instituição: UFU

Líder(es): Enivalda Nunes Freitas e Souza, Elzimar Fernanda Nunes Ribeiro Área: Letras

64. Poéticas do Imaginário e Memória. Instituição: UNIOESTE Líder(es): Antonio Donizeti da Cruz, Maria de Fatima Gonçalves Lima Área: Letras

65. Práticas culturais, religiosidade e imaginário. Instituição: UFRB Líder(es): Marco Antônio Nunes da Silva, Fabricio Lyrio Santos Área: História

66. Relações Raciais: Memória, Identidade e Imaginário. Instituição: PUC/SP Líder(es): Teresinha Bernardo, Regimeire Oliveira Maciel Área: Antropologia

67. Representação, Imaginário e Educação. Instituição: UFF Líder(es): Mary Rangel, Marcio Rodrigo Vale Caetano Área: Educação

68. Representações Sociais, Imaginário e Educação Contemporânea. Instituição: UNEB Líder(es): Paulo Batista Machado, Suzzana Alice Lima Almeida Área: Educação

69. RITe - Representações: Imaginário e Tecnologia. Instituição: USP Líder(es): Artur Simões Rozestraten, Karina Oliveira Leitão Área: Arquitetura e Urbanismo

70. Semiótica e construções identitárias: a semiotização da herança cultural árabeandaluza na geração de sentidos de identidade e alteridade no imaginário brasileiro. Instituição: UAM

Líder(es): Ricardo Nogueira de Castro Monteiro Área: Lingüística

71. Signo, imaginário, cultura. Instituição: UFBA Líder(es): Ana Rosa Neves Ramos, Cláudio José Meneses de Oliveira 
Área: Letras

72. Som, imagem e imaginário. Instituição: UNIBRASIL

Líder(es): Marcelo Eduardo Ribaric

Área: Comunicação

73. Symbolismum - Estudos Sobre Imaginário e Complexidade. Instituição: UFPE Líder(es): Eduardo Romero Lopes Barbosa

Área: Artes

74. Topos: Grupo de Estudos, Pesquisa e Extensão em Design, Educação e Imaginário Instituição: IFSUL

Líder(es): Alexandre Vergínio Assunção

Área: Desenho Industrial

75. Viagem, imaginários e socialidade. Instituição: UFJF

Líder(es): Euler David de Siqueira, Vera Maria Guimarães

Área: Turismo

76. Videlicet - Estudos em Intolerância, Diversidade e Imaginário. Instituição: UFPB Líder(es): Carlos André Macêdo Cavalcanti, Francisco de Assis Vale Cavalcante Filho Área: Teologia

77. Laboratório de Pesquisas Transdisciplinares sobre Moda. Instituição: UFPE Líder(es): Mario de Faria Carvalho, Allene Carvalho Lage Área: Desenho Industrial

78. Imaginário do morar contemporâneo. Instituição: UVA/RJ

Líder(es): Nara Pinto Iwata

Área: Arquitetura e Urbanismo

\section{Síntese das áreas de pesquisa:}

Letras/Linguística 19

Educação 16

Antropologia 15

Comunicação 10

Artes 9 
D. P. Rocha Pitta

Historia 6

Arquitetura/Urbanismo 3

Teologia 3

Filosofia 2

Enfermagem 2

Desenho industrial 2

Psicologia 1

Geografia 1

Turismo 1

Informação 1

(alguns grupos estão simultaneamente em 2 disciplinas)

Artigo recebido em: 30/07/2018

Artigo aprovado em: 31/08/2018 